\title{
Klein-Gordon Formulation of X-ray Diffraction in the Laue Case
}

\author{
J. BOROWSKI \\ Institute of Experimental Physics, University of Warsaw \\ Hoża 69, 00-681 Warsaw, Poland
}

\begin{abstract}
The Takagi-Taupin equations, the fundamental equations for X-ray diffraction deduced from the Maxwell equations, are considered. The connection between the Takagi-Taupin equations and the Klein-Gordon equation is shown. A method of solution of these equations using external differential form formalism is proposed. The solutions for both a narrow and a wide incident beams as a function of boundary conditions is analyzed. The so-called spherical and quasi-plane waves used in the X-ray experimental methods are derived from.
\end{abstract}

PACS numbers: $61.10 . \mathrm{Dp}, 02.30 . \mathrm{Jr}, 61.72 .-\mathrm{y}$

\section{Introduction}

In the present paper the Klein-Gordon formulation of the Takagi-Taupin equations (TTE) is described. The TTE, having the form of a set of two partial differential equations for two independent functions, have been derived by Takagi and Taupin [1, 2] for the electromagnetic fields in crystals from the Maxwell equations. The TTE are partial differential equations of the hyperbolic type. These equations can be converted to the Klein-Gordon equation (KGE) [3] describing the scalar field with a mass in relativistic quantum field theory. Naturally solutions of the KGE are well known and for this reason solutions of the TTE in the Laue [4] geometry have been presented earlier [5]. However, the present way of obtaining these solutions is very convenient for further applications for two reasons. Firstly, the use of external differential forms formalism and the generalized Stokes theorem [6] make the derivation of solutions clearer. This is very useful in the Laue case and even more in the Bragg case [4], where the mixed boundary conditions make the solutions much more complicated. Secondly, the formulation in the Klein-Gordon form gives the possibility to use the very well-known mathematical 
methods connected with the KGE. This is very useful for a description of diffuse scattering caused by microdefects [7], where their presence requires perturbation calculations [8]. The formulae presented in this work are valid for ideal crystals but the same formulae can give solutions for nonideal crystals in the zeroth order of perturbation calculations [9]. One cannot further reduce the amount of mathematical formulae, because this could lead to a loss of mathematical clarity, but all these formulae are quite simple and straightforward. On the other hand, the full final formulae used for the simulations are given below and can be directly applied, e.g. in the Mathematica computer package.

The solution of the TTE for both a narrow and wide incident beam are carefully analyzed in this work. Narrow beams are commonly used in the section topography experiments [10-12]. Their description based on the conventional X-ray dynamical theory $[14,15]$ is compared to the appropriate solutions of the TTE. Formation of the so-called quasi-plane wave on the base of solution of the TTE is also presented. This type of solutions (quasi-plane waves) are commonly used in investigations of microdefects [7] and for many other problems.

\section{Takagi-Taupin equations}

Let us consider the diffraction of an X-ray beam by a crystal of parallelepiped shape. For a given reflection vector $h$, which is orthogonal to the reflecting atomic planes, the following two complex vectors $\boldsymbol{k}_{0}$ and $\boldsymbol{k}_{h}$, with $\boldsymbol{k}_{h}=\boldsymbol{k}_{0}+\boldsymbol{h}$, are defined.

- The real parts of these vectors are lying in the plane formed by $h$ and the wave vector $\boldsymbol{K}_{e}$ of the incident beam. This plane is usually called the diffraction plane (plane of Fig. 1).

- The vectors $\boldsymbol{k}_{0}$ and $\boldsymbol{k}_{b}$ make the Bragg angle $\theta_{\mathrm{B}}$ with the reflecting planes, and $\left|\boldsymbol{k}_{0}\right|=\left|\boldsymbol{k}_{h}\right|=\frac{\nu_{0}}{c}\left[1+\frac{1}{2} \operatorname{Re}\left(\chi_{0}\right)\right], K=\left|\boldsymbol{K}_{e}\right|=\frac{\nu_{0}}{c}=\frac{1}{\lambda}$, where $\nu_{0}$ is the frequency of the incident wave, $c$ - the velocity of light in the vacuum, $\chi_{0}$ - the zeroth Fourier coefficient of the electric susceptibility of the crystal, and $\lambda$ - the wavelength of the incident beam. The direction of $k_{0}$ corresponds to the Bragg maximum in the kinematical diffraction theory [4].

- The imaginary parts $\boldsymbol{k}_{\mathrm{I}}$ of $\boldsymbol{k}_{0}$ and $\boldsymbol{k}_{h}$ are equal and defined as follows: $\left|\boldsymbol{k}_{\mathrm{I}}\right|=$ $\frac{\nu_{0}}{c} \frac{\left|\operatorname{Im}\left(\chi_{0}\right)\right|}{2 \cos \theta_{\mathrm{B}}} ; \boldsymbol{k}_{\mathrm{I}}$ is parallel to the reflecting planes.

Two co-ordinate systems $(x, z)$ and $(u, v)$, are used in the calculations (Fig. 1). The $x$-axis is lying in the entrance surface of the crystal and the $z$-axis is orthogonal to it. The directions of the $u$ - and $v$-axes are parallel to the directions of $\operatorname{Re}\left[\boldsymbol{k}_{0}\right]$ and $\operatorname{Re}\left[\boldsymbol{k}_{h}\right]$, respectively ( $u$ and $v$ are usually denoted by $s_{0}$ and $s_{h}$, respectively). Simple geometric considerations give the following transformation between $(x, z)$ and $(u, v)$ :

$$
\left(\begin{array}{l}
u \\
v
\end{array}\right)= \pm \frac{1}{\sin \left(2 \theta_{\mathrm{B}}\right)}\left(\begin{array}{cc}
C_{h}, & -S_{h} \\
-C_{0}, & S_{0}
\end{array}\right)\left(\begin{array}{l}
x \\
z
\end{array}\right)
$$


where $S_{j}:=\sin \left(\Psi_{j}\right), C_{j}:=\cos \left(\Psi_{j}\right), j=0, h$. The direction of the $x$-axis is chosen in such a way that always $\Psi_{0}>0$. The sign in (1) is to be taken positive when $\Psi_{0}>\Psi_{h}$, and negative otherwise.

The electric induction field in the crystal, as well as in the incident beam, is split into two polarization components, orthogonal to the diffraction plane and parallel to it. The differential equations for these two states of polarization differ only by a constant $C$ ( $C$ is usually called a polarization factor for the amplitude) $[1,2,4]$. The further considerations are given for a fixed polarization state, so the electric field is described by a scalar function.

The incident beam is given by the following formula:

$$
D_{e}(\boldsymbol{r})=A(\boldsymbol{r}) \exp \left(-2 \pi \mathrm{i} \boldsymbol{K}_{\mathrm{e}} \cdot \boldsymbol{r}\right)
$$

with $A(\boldsymbol{r})$ being its pseudo-amplitude.

The electric induction field, to be calculated in the crystal in the two-wave approximation $[2,4]$, is sought in the following form:

$$
d(\boldsymbol{r})=d_{0}(\boldsymbol{r}) \exp \left(-2 \pi \mathrm{i} \boldsymbol{k}_{0} \cdot \boldsymbol{r}\right)+d_{h}(\boldsymbol{r}) \exp \left(-2 \pi \mathrm{i} \boldsymbol{k}_{h} \cdot \boldsymbol{r}\right)
$$

where the pseudo-amplitudes $d_{j}(r)$ are slowly-varying functions, in contrast to the highly oscillating exponential terms. The Maxwell equations are applied to find the pseudo-amplitudes $d_{j}(r)$ but in the X-ray range some terms may be neglected and the final form of the differential equations is quite specific. Takagi's considerations lead to the following form of differential equations for the pseudo-amplitudes $d_{j}(r)$ in the ideal crystal:

$$
\partial_{0} d_{0}=-\mathrm{i} a d_{h}, \quad \partial_{h} d_{h}=-\mathrm{i} b d_{0},
$$

where $\partial_{j}:=\frac{\operatorname{Re}\left[\boldsymbol{k}_{j}\right]}{\left|\operatorname{Re}\left[\boldsymbol{k}_{j}\right]\right|}\left(\frac{\partial}{\partial x}, \frac{\partial}{\partial z}\right), a=\pi K C \chi_{\bar{h}}, b=\pi K C \chi_{h}$, and $\chi_{h}, \chi_{\bar{h}}$ - the Fourier coefficients of the electric susceptibility of the crystal.

Changing variables in the TTE (4) and introducing new symbols $\Phi_{u}$ and $\Phi_{v}$ for the pseudo-amplitudes

$$
d_{0}(x, z)=\Phi_{u}(u(x, z), v(x, z)), \quad d_{h}(x, z)=\Phi_{v}(u(x, z), v(x, z)),
$$

where $u(x, z)$ and $v(x, z)$ are given by $(1)$, one gets

$$
\begin{aligned}
& \partial_{u} \Phi_{u}=-\mathrm{i} a \Phi_{v}, \\
& \partial_{v} \Phi_{v}=-\mathrm{i} b \Phi_{u},
\end{aligned}
$$

where $\partial_{\sigma}:=\frac{\partial}{\partial \sigma}, \sigma=u, v$. Going from the linear set (6) of differential equations to the second order differential equation for $\Phi_{u}$ or $\Phi_{v}$ and setting

$$
\Phi(u, v)=: W(u+v, u-v)=W(t, y),
$$

where $\Phi$ is $\Phi_{u}$ or $\Phi_{v}$, the $\mathrm{KGE}[3]$

$$
\left(\partial_{t}^{2}-\partial_{y}^{2}+a b\right) W=0
$$

for function $W$ is derived. In this way, both $\Phi_{u}$ and $\Phi_{v}$ are obtained by solving the same KGE (8). The explicit formulae for $\Phi_{u}$ and $\Phi_{v}$ differ only, because different boundary conditions have to be used for these fields. 


\section{Solutions for the Laue geometry}

In the Laue geometry the incident beam is falling on one surface of the parallelepiped crystal (Fig. 1) and the diffracted beam, propagating along the $v$-axis, is emitted from the opposite surface (the lower side in Fig. 1). The electric induction of the incident beam is assumed to have the following form:

$$
D_{e}(r)=A(w) \exp \left(-2 \pi \mathrm{i} \boldsymbol{K}_{e} \cdot \boldsymbol{r}\right),
$$

where $w$ is co-ordinate across the beam (Fig. 2). In order to simulate the usually experimental conditions, the function $A(w)$ is taken in the following form:

$$
A(w)=f_{k, l}(w-l-k),
$$

where for positive arguments $x$

$$
f_{k, l}(x)= \begin{cases}0 & x \geq l+k, \\ \exp \left[-\frac{(x-l)^{2}}{k^{2}-(x-l)^{2}}\right], & l<x<l+k, \\ 1, & x \leq l,\end{cases}
$$

and $f(-x):=f(x)$. This is an analytical function with connected support [6]. In the case when $k \ll l$ this function is very close to the non-smooth characteristic function (the step function) of the area $[0,2 l+2 k]$ but for all values of the parameters $l$ and $k$ it is analytical.

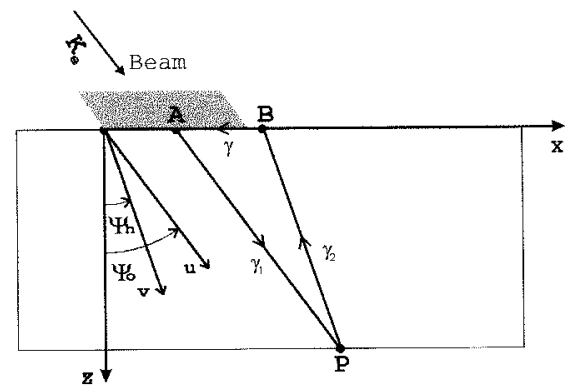

Fig. 1. Geometry of diffraction. The X-ray beam (shadow area) is falling on the crystal (rectangle) along the $\boldsymbol{K}_{e}$ direction. The fields inside the investigated crystal are obtained using the co-ordinates $(x, z)$ and $(u, v)$. For a given point $P$ the curve $\gamma$ is lying on the entrance surface $S$ of the crystal between the characteristic lines $\left(\gamma_{1}\right.$ and $\left.\gamma_{2}\right)$ parallel to the $u$ and $v$ directions, respectively, and meeting in $P$. The fields in the point $P$ depend linearly on the field of the incident beam on the curve $\gamma$. If $P$ takes such a place that all of $\gamma$ is lying outside the illuminated (shadowed) area then the field in $P$ vanishes.

It follows from the Maxwell equations the continuity of the electric induction $D$ on the entrance surface: the $D$ component normal to the surface is continuous but for X-ray radiation the refraction coefficient is very close to unity so that also the tangential component of the induction is to a very good approximation 


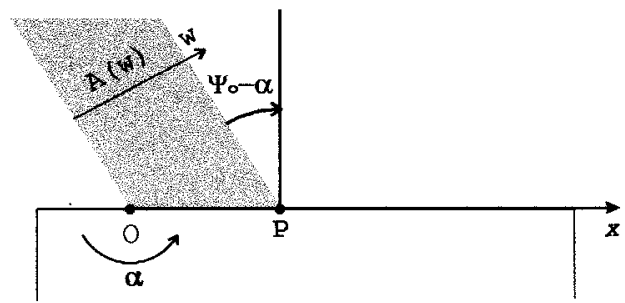

Fig. 2. Geometry of the boundary condition (12). The X-ray beam bounded in space (shadowed area) is falling on the crystal of Fig. 1. The electric induction field of the incident beam is given by the analytical function $A$ of the co-ordinate $w$ across the beam (Fig. 3). The crystal can be rotated around $O$ (the left edge of the illuminated area). The point $O$ has co-ordinate $x=0$ for any value of the rotation angle $\alpha$ but the $x$ co-ordinate of the right boundary (point $P$ ) depends on $\alpha$.

continuous. Comparing (2) to (3) on the entrance surface $S(z=0)$ and taking into account that $\left|k_{0}\right|=\left|\boldsymbol{K}_{e}\right|\left(1+\frac{1}{2}\left|\chi_{0}\right|\right)$ one gets

$$
\begin{aligned}
& D_{\left.\epsilon\right|_{S}}(\boldsymbol{r}) \exp \left[2 \pi \mathrm{i} K \cos \left(\Psi_{0}\right)\left(\alpha-\alpha_{0}\right) x\right]-d_{0}(x, 0) \\
& \quad=d_{h}(x, 0) \exp (-2 \pi \mathrm{i} \boldsymbol{h} \cdot \boldsymbol{r})_{\left.\right|_{S}},
\end{aligned}
$$

where $\alpha_{0}=\frac{1}{2}\left|\chi_{0}\right| \tan \Psi_{0}$, and $\alpha$ is the angle of rotation of the crystal along a line orthogonal to the plane of Fig. 2 and going through $O$. The left side of (12) is a slowly varying function of $x$ for small values of $\alpha-\alpha_{0}$. On the contrary, the right side of (12) contains the highly oscillatory term $\exp (-2 \pi \mathrm{i} h \cdot r)$. For this reason formula (12) can be fulfilled only when both sides of (12) vanish. In this way, from the Maxwell equations alone, the boundary conditions for the TTE are

$$
d_{0}(x, 0)=A\left[x \cos \left(\Psi_{0}-\alpha\right)\right] \exp \left[2 \pi \mathrm{i} K \cos \left(\Psi_{0}\right)\left(\alpha-\alpha_{0}\right) x\right]
$$

and

$$
d_{h}(x, 0)=0 .
$$

From (6a), (13), and (14) it follows:

$$
\partial_{u} \Phi_{\left.u\right|_{S}}=0
$$

and

$$
\Phi_{\left.u\right|_{S}}=d_{0}(x, 0) .
$$

Equations (13), (14), and (6b) give the boundary conditions for $\Phi_{\imath}$

$$
\Phi_{\left.v\right|_{s}}=0
$$

and

$$
\partial_{v} \Phi_{\left.v\right|_{s}}=-\mathrm{i} b d_{0}(x, 0) .
$$

From (6) it follows that $\Phi_{u}$ as well as $\Phi_{v}$ fulfil the same differential equation 


$$
\left(\partial_{u} \partial_{v}+a b\right) \Phi=0
$$

which is to be solved under boundary conditions (15) and (16) for the field $\Phi_{u}$ or (17) and (18) for the field $\Phi_{v}$. In order to find the fields $\Phi$ in every point $P$ lying in the crystal or on its exit surface (Fig. 1) the following function $R_{P}$ is defined:

$$
R_{P}(u, v):=J_{0}\left[2 \sqrt{a b} \sqrt{\left(u_{P}-u\right)\left(v_{P}-v\right)}\right],
$$

where $\left(u_{P}, v_{P}\right)$ stands for co-ordinates of the point $P$. For a given $P$ the area of the triangle $P B A$ is considered. The line $A P\left(\right.$ or $\left.\gamma_{1}\right)$ is parallel to the $u$-direction and $P B$ (or $\left.\gamma_{2}\right)$ - to the $v$-direction. For every point $(u, v)$ within the triangle $P B A$ the value of $\left(u_{P}-u\right)\left(v_{P}-v\right)$ under square root in (20) is positive. It may be verified that the function defined in (20) satisfies (19) and the boundary conditions

$$
\left.\partial_{u} R_{P}\right|_{\gamma_{1}} \equiv 0,\left.\quad \partial_{v} R_{P}\right|_{\gamma_{2}} \equiv 0 .
$$

In order to find $\Phi_{u}$ and $\Phi_{v}$ in $P$ two external differential forms [6] are defined

$$
\Omega_{u}:=-\left(R_{P} \partial_{u} \Phi_{u}\right) \mathrm{d} u-\left(\Phi_{u} \partial_{v} R_{P}\right) \mathrm{d} v
$$

and

$$
\Omega_{v}:=\left(\Phi_{v} \partial_{u} R_{P}\right) \mathrm{d} u+\left(R_{P} \partial_{v} \Phi_{v}\right) \mathrm{d} v .
$$

From the definitions and the fact that $\left(\partial_{u} \partial_{v}+a b\right) R_{P} \equiv 0$ it follows that the external derivative [6] for both these forms vanishes

$$
\mathrm{d} \Omega=0 .
$$

Applying the Stokes theorem stating the integral of the external derivative of a differential form over a given area is equal to the integral of this form over the boundary of this area, i.e.

$$
0=\int_{P B A} \mathrm{~d} \Omega=\int_{\gamma_{1} \cup \gamma_{2} \cup \gamma_{3}} \Omega
$$

and involving Eqs. (21) one gets

$$
-\int_{\gamma_{1}}\left(\partial_{u} \Phi_{u}\right) \mathrm{d} u-\int_{\gamma}\left[\left(R_{P} \partial_{u} \Phi_{u}\right) \mathrm{d} u+\left(\Phi_{u} \partial_{v} R_{P}\right) \mathrm{d} v\right]=0
$$

and

$$
\int_{\gamma_{2}}\left(\partial_{v} \Phi_{v}\right) \mathrm{d} v+\int_{\gamma}\left[\left(\Phi_{v} \partial_{u} R_{P}\right) \mathrm{d} u+\left(R_{P} \partial_{v} \Phi_{v}\right) \mathrm{d} v\right]=0,
$$

where $\gamma$ is oriented curve from $B$ to $A$ (Fig. 1). Taking into account that $\int_{\gamma_{1}}\left(\partial_{u} \Phi_{u}\right) \mathrm{d} u=\Phi_{u}\left(u_{A}, v_{A}\right)-\Phi_{u}\left(u_{P}, v_{P}\right)$ and using (6), from (26) one gets

$$
\Phi_{u}\left(u_{P}, v_{P}\right)=\Phi_{u}\left(u_{A}, v_{A}\right)-\int_{\gamma}\left(\Phi_{u} \partial_{v} R_{P}\right) \mathrm{d} v .
$$

In the same way the following expression for the field $\Phi_{v}$ is obtained:

$$
\Phi_{v}\left(u_{P}, v_{P}\right)=\Phi_{v}\left(u_{B}, v_{B}\right)-\int_{\gamma} \mathrm{i} b R_{P} \Phi_{u} \mathrm{~d} v
$$


From (26) and (27) it follows that if for a given point $P$ the curve $\gamma$ is lying on the non-illuminated area of the entrance crystal surface then the fields $\Phi$ vanish in $P$. This is a general feature of partial differential equations of the hyperbolic type: the fields propagate only between characteristic lines.

\section{Solutions for a small width of the incident beam}

The case when the width of the incident beam is much smaller than the length $|B A|$ of $\gamma$ (Fig. 1) is common in section topography arrangements [10-12]. From (1) it follows that the dependence of the co-ordinates $(u, v)$ on the $x$ (Fig. 1) for $\gamma$ has the form:

$$
\gamma:[0, \epsilon] \ni x^{\prime} \longmapsto \gamma\left(x^{\prime}\right)=\left(\begin{array}{c}
u \\
v
\end{array}\right)= \pm \frac{1}{\sin \left(2 \theta_{\mathrm{B}}\right)}\left(\begin{array}{c}
C_{h} x^{\prime} \\
-C_{0} x^{\prime}
\end{array}\right)
$$

and

$$
\left(\begin{array}{c}
u_{P}-u \\
v_{P}-v
\end{array}\right)= \pm \frac{1}{\sin \left(2 \theta_{\mathrm{B}}\right)}\left(\begin{array}{c}
C_{h}\left(x-x^{\prime}\right)-S_{h} T \\
-C_{0}\left(x-x^{\prime}\right)+S_{0} T
\end{array}\right) .
$$

Setting in (13) $\alpha=\alpha_{0}$ (the dependence of $d_{h}$ on the $\alpha$ was analysed in [13]) we have for $d_{0}$ on the entrance surface

$$
d_{0}\left(x^{\prime}, 0\right)=A\left[x^{\prime} \cos \left(\Psi_{0}-\alpha_{0}\right)\right]
$$

for $x^{\prime} \in[0, \epsilon]$ and 0 outside this region, where $\epsilon=\frac{2(l+k)}{\cos \left(\Psi_{0}-\alpha\right)}$. Applying (30), (31), and (32) to (29) and then using (5), one gets the expression for $d_{h}$

$$
d_{h}(x, T)=-\mathrm{i} b \frac{C_{0}}{\sin \left(2 \theta_{\mathrm{B}}\right)} \int_{0}^{\epsilon} \mathrm{d} x^{\prime} J_{0}(2 \sqrt{a b} \sqrt{q})\left\{A\left[x^{\prime} \cos \left(\Psi_{0}-\alpha_{0}\right)\right]\right\},
$$

where $q=\left(u_{P}-u\right)\left(v_{P}-v\right)$ is a function of $x^{\prime}$ given by (31). Changing the variable in (33) to $y=\frac{1}{2 C_{0} C_{h}}+\frac{x-T \tan \Psi_{j}-x^{\prime}}{T \sin \left(2 \theta_{\mathrm{B}}\right)}$, where $\Psi_{j}=\Psi_{0}$ for plus in (1) and $\Psi_{j}=\Psi_{h}$ for minus in (1), one gets

$$
\begin{aligned}
& d_{h}(x, T)=\mathrm{i} b C_{0} T \int_{y_{1}}^{y_{1}-\frac{\epsilon}{T \sin \left(2 \theta_{\mathrm{B}}\right)}} \mathrm{d} y J_{0}\left(2 \sqrt{a b} T \sqrt{C_{0} C_{h}} \sqrt{\frac{1}{\left(2 C_{0} C_{h}\right)^{2}}-y^{2}}\right) \\
& \quad \times\left.\left\{A\left[x^{\prime} \cos \left(\Psi_{0}-\alpha_{0}\right)\right]\right\}\right|_{x^{\prime}=x^{\prime}(y)},
\end{aligned}
$$

where $y_{1}=\frac{1}{2 C_{0} C_{h}}+\frac{x-T \tan \Psi_{j}}{T \sin \left(2 \theta_{\mathrm{B}}\right)}$. In the limiting case when $\epsilon$ is very small, the integral in (33) can be changed to $\frac{-\epsilon}{T \sin \left(2 \theta_{\mathrm{B}}\right)}$ multiplied by the value of the integrand in $y_{1}$ and the approximate formula takes the following form:

$$
d_{h}(x, T)=-\mathrm{i} b \frac{\epsilon C_{0}}{\sin \left(2 \theta_{\mathrm{B}}\right)} T J_{0}\left(\frac{2 \sqrt{a b} T}{\sqrt{C_{0} C_{h}}} \sqrt{1-\frac{s^{2}}{L^{2}}}\right),
$$

where $s=x-\frac{T}{2}\left(\tan \Psi_{0}+\tan \Psi_{h}\right), L=\frac{T}{2} \frac{\sin \left(2 \theta_{\mathrm{B}}\right)}{C_{0} C_{h}}$. In order to obtain (35) $A\left(y_{1}\right)=1$ 
was set. The limiting formula (35) was first obtained by Kato [14, 15] using quite different considerations, and then by Takagi [5]. Both not contain the factor $\epsilon$ describing the dependence of the emitted field on the width of the incident beam. The presence of factor $\epsilon$ in (35) expresses the energy conservation law in the case of a fully coherent incident beam: the total energy of the incident beam is proportional to $\epsilon^{2}$ and the emitted beams must transport all this energy. The dependency of $d_{b}$ on $\epsilon$ in (35) can be used to investigate a degree of coherence of the incident beam because for the incoherent or partly coherent beam the dependence on $\epsilon$ in (35) will take another form [16].

Formula (34) was directly applied to obtain the diffracted amplitude $d_{h}$ for a silicon crystal set for the reflection $h=[333], \theta_{\mathrm{B}}=19.84^{\circ}$. For the surface

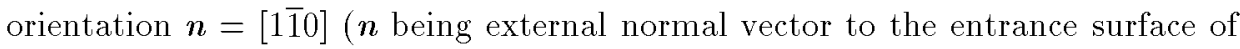
the crystal) the values of the angles $\Psi$ are: $\Psi_{0}=55.1^{\circ}, \Psi_{h}=15.4^{\circ}$. The thickness of the crystal was taken as $T=270 \mu \mathrm{m}, C=1$ and the wavelength was taken for the $\mathrm{Cu} K_{\alpha_{1}}$ radiation. The function $A$ for $l=20 \mu \mathrm{m}$ and $k=0.02 \mu \mathrm{m}$ (full line), $l=15.02 \mu \mathrm{m}$ and $k=5 \mu \mathrm{m}$ (broken line), $l=5.02 \mu \mathrm{m}$ and $k=15 \mu \mathrm{m}$ (dotted line) taken as input condition (32) is presented in Fig. 3. The full width of the incident beam is taken to be equal to $40.04 \mu \mathrm{m}$ for all these values of $l$ and $k$. For the first pair of the parameters this function is very close to the characteristic function but is an analytical one. This fact is important to exclude the hypothesis



Fig. 3

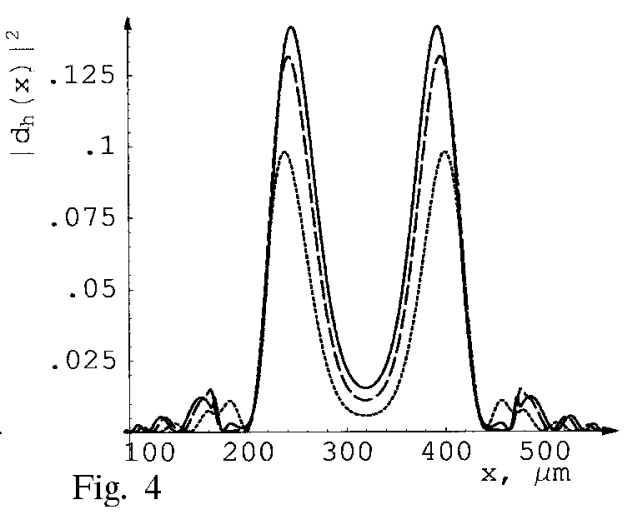

Fig. 4

Fig. 3. Pseudo-amplitude $A$ of the incident electric induction, taken as boundary condition for the simulations presented in Fig. 4 and plotted as a function of the co-ordinate $w$ across the beam. Changing the parameters $l$ and $k$ one gets different shapes of $A$ (see description in the text). The three pairs of parameters $l$ and $k$ are chosen in such a way that for every pair the full width of the beam is $40.04 \mu \mathrm{m}$. Even for the full line, which looks like a step-function, $A$ is an analytical function.

Fig. 4. The diffracted wave $\left|d_{h}\right|^{2}$ as a function of the $x$ co-ordinate along the exit surface of the crystal. The amplitude $d_{h}$ was obtained numerically using (34). The three lines correspond to the three boundary conditions taken (Fig. 3). 
that oscillations arising near the borders of the emitted field $d_{h}$ (Fig. 4) are caused by noncontinuous boundary conditions.

The squared module of the diffracted beam $\left|d_{h}\right|^{2}$ as a function of the co-ordinate $x$ (Fig. 1) on the exit surface of the crystal is shown in Fig. 4. The three lines correspond to the boundary conditions taken for the simulations (Fig. 3). The two peaks in the middle of Fig. 4 have different heights depending on the boundary condition. The maximal value here is for the full line and minimal for the dotted one. This is due to the fact that $d_{h}$ depends on the boundary condition linearly and the area under the full line is greater than under the other lines. However, the structure of the border peaks in Fig. 4 is much more complicated due to the multiplication of the two functions in the integrand of (34). It is clear that the emitted field $d_{h}$ strongly depends on the boundary condition and doing simulations for a given experimental set-up one should first measure the profile of the incident beam.

The scaling of the co-ordinate axis for all simulations of $d_{h}$ is determined by $A$ (the maximal value of $A$ is set to one but in a real experiment this value should be measured). The function $\left|d_{h}\right|^{2}$ of $x$ for $A$ having a form very close to the characteristic function ( $k=0.02 \mu \mathrm{m}$ ) was simulated using (34) for three different values of $l$ and is plotted in Fig. $5: l=1 \mu \mathrm{m}$ (full line), $l=2 \mu \mathrm{m}$ (broken line) and $l=4 \mu \mathrm{m}$ (dotted line). From the values for the third peak on the left side of Fig. 5: 0.001 (full), 0.004 (broken), and 0.014 (dotted) it is seen that the linear approximation (35) is valid for the full and broken lines but not for the dotted one. The latter one is for the width of slit set to $8.04 \mu \mathrm{m}$ in a section topography (the usual experimental width of the slit) and for this case or for wider slits (34) should be applied.

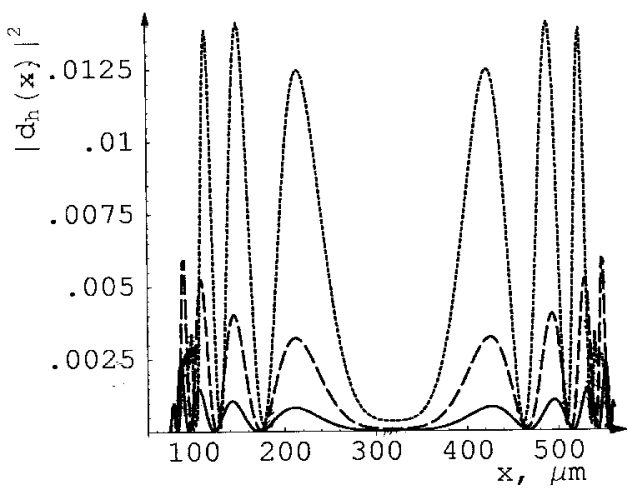

Fig. 5. The diffracted wave $\left|d_{h}\right|^{2}$ as a function of the $x$ co-ordinate on the exit surface for different widths of the incident beam: $2.04 \mu \mathrm{m}$ (full line), $4.04 \mu \mathrm{m}$ (broken line), and $8.04 \mu \mathrm{m}$ (dotted line). 


\section{Solution for a wide beam}

In experimental arrangements where the incident beam is much wider than $|B A|$ (Fig. 1) so-called quasi-plane waves (often called in the X-ray jargon also simply plane waves) are formed. The Fourier transform analysis of beams emitted by crystals containing randomly distributed microdefects can then be performed [7]. First, we consider the simplest case when $P$ is in such a place that all of $B A$ is lying in the part of illuminated area where $A=1$. Contrary to Sec. 4 , the $\Delta \alpha=\alpha-\alpha_{0}$ is treated as an independent variable (not set to zero) and the question is how the field in $P$ depends on $\Delta \alpha$. In this case formula (32) for the field $d_{0}$ on the entrance crystal surface takes the following form:

$$
d_{0}(x, 0)=\exp \left[-2 \pi \mathrm{i} K \cos \left(\Psi_{0}\right) \Delta \alpha x^{\prime}\right]
$$

and formulae (30) and (31) are still valid. Formula (34) can be applied to find the field in $P$ but with two changes. Firstly, the limits in (34) are changed to $\mp \frac{1}{2 C_{0} C_{h}}$, because the limits in (33) are now $x-T \tan \Psi_{h}$ (lower) and $x-T \tan \Psi_{0}$ (upper). Secondly, the square-bracketed term in the integrand (coming from the boundary condition) must now be changed to the right side of (36). Changing the variable in $(34)$ as before we have for the field in $P$

$$
\begin{aligned}
& d_{h}(x, T)=-\mathrm{i} b C_{0} T \exp \left\{2 \pi \mathrm{i} K C_{0} \Delta \alpha\left[x-\frac{T}{2}\left(\tan \Psi_{0}+\tan \Psi_{h}\right)\right]\right\} \int_{-\frac{1}{2 C_{0} C_{h}}}^{\frac{1}{2 C_{0} C_{h}}} \mathrm{~d} y \\
& \times \exp \left[\mp 2 \pi \mathrm{i} K C_{0} \Delta \alpha T \sin \left(2 \theta_{\mathrm{B}}\right) y\right] J_{0}\left(2 \sqrt{a b} \sqrt{C_{0} C_{h}} T \sqrt{\frac{1}{\left(2 C_{0} C_{h}\right)^{2}-y^{2}}}\right),
\end{aligned}
$$

where the sign under the exponential function is positive if the sign in (1) is negative, and vice versa. Taking into account that $\int_{-a}^{a} \exp (i u) J_{0}\left(v \sqrt{a^{2}-x^{2}}\right) \mathrm{d} x=$ $2 \frac{\sin \left(a \sqrt{u^{2}+v^{2}}\right)}{\sqrt{u^{2}+v^{2}}}[17]$, the integration in (37) can be done and one obtains for $d_{h}$

$$
\begin{gathered}
d_{h}(x, T)=-\mathrm{i} \sqrt{\frac{C_{0}}{C_{h}}} \exp \left\{2 \pi \mathrm{i} K C_{0} \Delta \alpha\left[x-\frac{T}{2}\left(\tan \Psi_{0}+\tan \Psi_{h}\right)\right]\right\} \\
\times \frac{\chi_{h}}{\sqrt{\chi_{h} \chi_{\bar{h}}}} \frac{\sin \left(\frac{\pi K T C \sqrt{\chi_{h} \chi_{\bar{h}}}}{\sqrt{C_{0} C_{h}}} \sqrt{1+r^{2}}\right)}{\sqrt{1+r^{2}}},
\end{gathered}
$$

where $r=\sqrt{\frac{C_{0}}{C_{h}}} \frac{\sin \left(2 \theta_{\mathrm{B}}\right) \Delta \alpha}{C \sqrt{\chi_{h} \chi_{\bar{h}}}}$. Expression (38) was first derived from the TTE by Takagi himself [5] and it agrees well with the formula obtained in the conventional $\mathrm{X}$-ray dynamical theory [4] assuming an incident beam in the form of an infinite plane wave (non-physical incident beam).

The real part of the diffracted amplitude $d_{h}$ was directly obtained from (37) and plotted as a function of $\Delta \alpha$ in Fig. 6. The curve in Fig. 6 can also be obtained analytically using (38). The numerical results from Fig. 6 are compared to the 


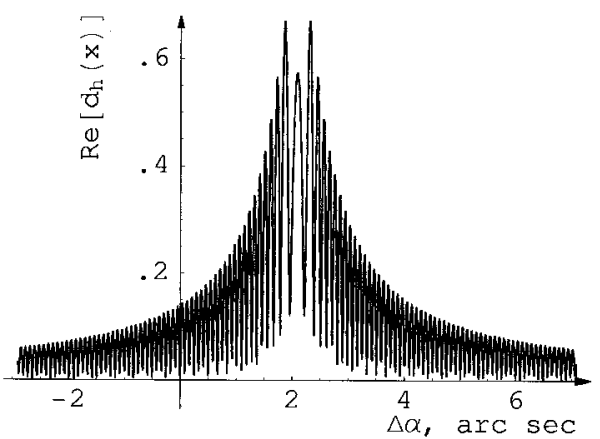

Fig. 6. The real part of the pseudo-amplitude of the diffracted wave $\operatorname{Re}\left(d_{h}\right)$ as a function of $\Delta \alpha$. The incident beam is so wide that a point $P$ (Fig. 1) can be found for which all of $\gamma$ is lying in the part of the illuminated area where $A=1$. For this point the field $d_{h}$ was obtained numerically using (37).

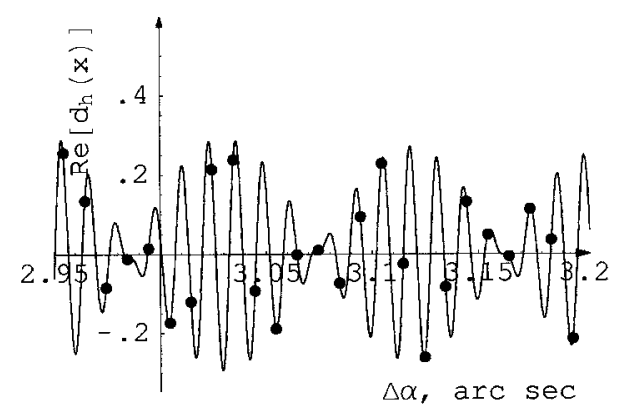

Fig. 7. The test of the computer program used for simulations. The rocking curve obtained numerically (dots) in Fig. 6 from (37) is compared to the results obtained analytically using (38) (solid line).

analytical ones in Fig. 7. Here, the numerical points (dots) from a part of Fig. 6 are shown on along with the analytical curve (solid line) and it is clearly seen that the agreement is excellent.

Returning to the spatial dependence of $d_{h}$ in the case of a wide incident beam, $\Delta \alpha$ was set again to zero. As shown above (formula (37) or (38)), in the case when $\gamma$ is lying in the part of the illuminated area where the amplitude of the incident beam is constant, $\left|d_{h}\right|$ does not depend on the position $x$. In this way, for $x \in\left[T \tan \left(\Psi_{h}\right), \epsilon+T \tan \left(\Psi_{0}\right)\right]$ the squared module of $d_{h}$ is the constant given by (38). Changing the observation point $P$ to the position for which the curve $B A$ (Fig. 1) is lying partly on the area of the nonconstant amplitude of the incident beam (Fig. 3), $\left|d_{h}\right|$ does depend on $x$ and can be obtained numerically using (37). The dependence of $\left|d_{h}\right|^{2}$ on $x$ is shown in Fig. 8 . Here $l=150 \mu \mathrm{m}$ and $k=0.02 \mu \mathrm{m}$ was used in the simulation; $\epsilon$ is a function of $l$ and $k$ as above. In the case when 


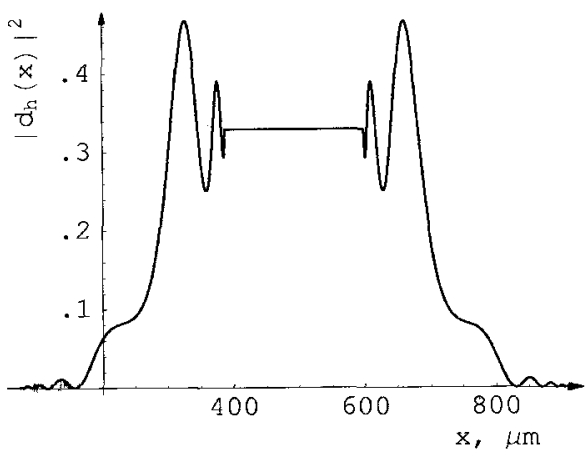

Fig. 8. The dependence of the diffracted wave $\left|d_{h}\right|^{2}$ on the co-ordinate $x$ (in $\mu \mathrm{m}$ ) on the exit surface; $d_{h}$ was obtained directly from (37).

the length of the constant area in Fig. 8 is much greater than the lengths of the oscillatory border parts, the emitted wave $d_{h}$ is usually called quasi-plane wave in the $\mathrm{X}$-ray applications.

\section{Discussion}

A careful description of the mathematical structure of the TTE was done in Sec. 2. The connection of these equations with the well-known KGE gives the possibility to use the methods known for the latter. The TTE in the form similar to (8) or even in the form of an elliptical differential equation were investigated long ago by Russian theoreticians Indenboom and Chukhovskii [17], however without referring to the KGE. The analytical expressions given in this work are valid for an ideal crystal but the same expressions, after changing the values of $\chi_{h}$ and $\chi_{\bar{h}}$, are applicable for a crystal containing statistically distributed microdefects in the zeroth order of perturbation calculations. The mathematical formalism presented here can be directly used to obtain higher terms of the perturbation calculations [8] for a nonideal crystal.

The application of external differential forms in Sec. 3 gives a very clear way to obtain solutions of TTE. It is especially important for considerations connected with diffraction in the Bragg geometry [4] (not presented here), which is much more complicated than the calculations in the Laue geometry. Using external differential forms one can very easily get an integral equation which is to be solved in the Bragg case. However, the solution of this integral equation is not straightforward and will be presented elsewhere.

Formula (35) for section topography was first obtained by Kato [14, 15] using the following consideration. A spherical wave was constructed from plane waves (Fourier transform) and this spherical wave was assumed to be falling on a crystal. For each plane-wave component of the spherical wave, the diffracted wave 
was obtained using the conventional X-ray dynamical theory as given by (38). Next, the solutions (38) were added (i.e. the Fourier transform was performed) to get expression (35) but without the term $\epsilon$ and without the dependence on the boundary condition.

The point of view presented in the present work is quite different: the TTE are solved for a narrow incident beam and expression (34) is obtained. Formula (34) depends on the boundary condition and the width of the slit. In the limit of a very narrow slit (and setting $A=1$ ) formula (35), given by Kato, is obtained. Similar considerations were presented by Takagi [5] (without the dependence on boundary condition) and they were also mentioned in Pinsker's monograph [17]. These two quite different points of view can probably be explained as follows. In section topography the incident beam is very narrow (but not a spherical beam) and using the conventional theory one should perform its Fourier transform. Probably the solutions of TTE for these two different Fourier transforms are similar and in the limiting case expression (35) is valid for both these descriptions. The dependence of these solutions on the Fourier transform used were analysed in [13]. The description based on the TTE presented in this work is much clearer than the one involving calculations within the conventional theory.

Also, the Green functions for the TTE $[18,19]$ can be easily derived from the solutions presented in this work.

\section{Conclusions}

The solutions of the TTE in the Laue case based on the external differential forms and the Stokes theorem were obtained. A careful analysis of the solutions for a narrow and a wide incident beams is presented to describe experimental methods applied in X-ray investigations. The importance of the state of coherence of the falling beam is stressed. The methods presented in this work will be applied for a description of X-ray diffraction in the Bragg case and for higher orders of perturbation calculations for crystal containing microdefects.

\section{Acknowledgments}

I would like to thank Professor M. Lefeld-Sosnowska for introducing me to the statistical dynamical X-ray diffraction theory and section topography basics, Professor J. Gronkowski for physical interpretation of the TTE and Dr. E. Zielińska-Rohozińska for introducing me to the diffuse scattering theory. Important discussions with Dr. G. Kowalski on advanced topics of X-ray diffraction are much appreciated. 


\section{References}

[1] S. Takagi, Acta Crystallogr. 15, 1311 (1962).

[2] S. Takagi, J. Phys. Soc. Jpn. 26, 1239 (1969).

[3] I. Bialynicki-Birula, Z. Bialynicka-Birula, Quantum Electrodynamics, Oxford University Press, Oxford 1970.

[4] W.H. Zachariasen, Theory of X-Ray Diffraction in Crystals, Willey \& Sons, New York 1945.

[5] S. Takagi, unpublished results.

[6] L. Schwartz, Analyse mathématique, Hermann, Paris 1970.

[7] J. Gronkowski, J. Borowski, E. Zielińska-Rohozińska, Phiplos. Trans. R. Soc. Lond. A 357, 2721 (1999).

[8] J. Borowski, Ph.D. Dissertation, University of Warsaw, Warsaw 1998 (in Polish).

[9] V. Holý, Phys. Status Solidi B 111, 341 (1982).

[10] A.R. Lang, Acta Metallurgica 5, 358 (1957).

[11] G. Kowalski, M. Lefeld-Sosnowska, J. Gronkowski, J. Borowski, Philos. Trans. R. Soc. Lond. A 357, 2707 (1999).

[12] J. Borowski, Comp. Materials Sci. 5, 286 (1996).

[13] J. Borowski, J. Gronkowski, J. Alloys Comp. 328, 211 (2001).

[14] N. Kato, Acta Crystallogr. 14, 526 (1961).

[15] N. Kato, Acta Crystallogr. A 36, 770 (1980).

[16] J. Borowski, J. Gronkowski, J. Phys. D, Appl. Phys. 34, 3496 (2001).

[17] Z.G. Pinsker, Dynamical Scattering of X-Rays in Crystals, Springer-Verlag, Berlin 1978.

[18] A. Authier, D. Simon, Acta Crystallogr. A 24, 517 (1968).

[19] A.M. Afanas'ev, V.G. Kohn, Acta Crystallogr. A 27, 421 (1971). 